\title{
CARACTERÍSTICAS DE PRESERVACIÓN DE CUATRO MADERAS DE BOSQUES SECUNDARIOS DE PUCALLPA
}

\author{
Leticia Guevara Salnicov *
}

\section{RESUMEN}

El Proyecto Estudios Tecnológicos de Maderas de Bosques Secundarios que ejecuta el Convenio IIAP-UNU ha estudiado algunas propiedades tecnológicas de cuatro maderas provenientes de un bosque secundario de Pucallpa, Ucayali. Uno de los ensayos efectuados es el de la determinación de las características de preservación por el método de inmersión prolongada con solución de pentaclorofenol en petróleo al 5\% a temperatura ambiente. Se utilizaron probetas de madera de Trema micrantha (atadijo), Sapiurn marmieri Hubert (caucho rnasha), Jacaranda copaia (Aubl.) D. Don (huamanzamana) y Ochroma piramydale Swartz (topa) de $2 \mathrm{~cm}$ x $2 \mathrm{~cm}$ x $40 \mathrm{~cm}$, libres de defectos y signos de biodeterioro, procedentes de tres árboles por especie y dos niveles por árbol. Se utilizó la metodología descrita por Aróstegui (1970). Los resultados obtenidos indican que tres maderas son fáciles de tratar y una es moderadamente tratable.

\section{ABSTRACT}

The Project of Technological Studies of Lumber of Secondary Forests undertaken by the IIAP-UNU Agreement has studied some technological properties of four kinds of wood from a secondary forest in Pucallpa, Ucayali. One of the papers determines the preservation features by the method of prolonged irnmersion with a pentaclorophenol solution in petroleurn at $5 \%$ at normal ternperature. $2 \mathrm{~cm} \times 2 \mathrm{~cm}$ x $40 \mathrm{~cm}$ wooden test tubes made of Trema micrantha (atadijo); Sapiurn marmieri Hubert (rnasha rubber); Jacavanda copaia (Aubl.) D. Don (huamanzamana) and Qchrorna piramydale Swartz (topa) were used; they were all fault-free and had no signs of biodeterioration. They carne from three different trees per species and two levels pertree. The methodology designed by Aróstegui et al. (1970) was used. The result indicate that three of the types of wood undergo treatment easily while one does moderately. 


\section{INTRODUCCION}

En los bosques amazónicos secundarios existe un gran potencial de recursos forestales, especialmente los maderables. Actualmente no son aprovechados debido, entre otros factores, al desconocimiento de sus propiedades tecnológicas y aptitud de uso.

Para lograr el aprovechamiento integral de estas áreas de producción, caracterizadas por la presencia de especies pioneras de rápido crecimiento, es indispensable el estudio de las propiedades tecnológicas de las especies potencialmente aprovechables. Una de estas propiedades particularmente importante de definir en especies de rápido crecimiento generalmente de baja durabilidad natural- y para asegurar su aceptación en el mercado, es el comportamiento al tratamiento preservador.

El presente trabajo informa sobre los resultados obtenidos en los ensayos de preservación por inmersión prolongada a temperatura ambiente en soluciones de pentaclorofenol efectuadas con cuatro maderas de especies forestales de bosques secundarios. Los ensayos e interpretación de resultados se hicieron de acuerdo con las especificaciones propuestas por Aróstegui (1970).

El trabajo se desarrolló dentro del marco del Proyecto Estudios Tecnológicos de Maderas de Bosques Secundarios que ejecuta el Convenio JJAP-UNU.

\section{REVISION DE LITERATURA.}

Según Gonzáles (1970), los factores que influyen en la retención y penetración son numerosos. Destacan por su importancia la densidad básica, anatomía de la madera y método de tratamiento. Hunt y Garrat (1962) añaden a estos factores la naturaleza del preservador, viscosidad y temperatura de la solución de tratamiento y la geometría de la pieza. Además, para la mayor parte de métodos de tratamiento, el contenido de humedad influye determinantemente en la capacidad de impregnación, profundidad y distribución del preservador. Por debajo del punto de saturación de la fibra, la solución de tratamiento puede fluir libremente a través de las cavidades celulares y migrar por difusión a las paredes celulares, aunque la difusión se verifica exclusivamente en preservadores hidrosolubles. 
La eficacia y la economía absoluta de un tratamiento se miden con la duración de la madera preservada. En la práctica el criterio que prevalece para evaluar la utilidad de un tratamiento es el volumen de solución absorbido y retenido por la madera y la profundidad de penetración.

No sólo los tratamientos industriales que requieren de equipos y maquinarias son eficaces. Según Hunt y Garrat (1962) la protección de la madera preservada por cualquier método de tratamiento depende fundamentalmente de la capacidad para conseguir una penetración profunda y uniforme. La cantidad de preservador finalmente retenido en la madera depende principalmente del uso al que se le destina.

El método de tratamiento por inmersión prolongada es adecuado para madera seca, porosa y con preservadores oleosolubles. En principio, de acuerdo con el tiempo de inmersión, la absorción y penetración pueden igualar a las que se obtienen por métodos a presión.

\section{MATERIALES Y METODOS}

\section{Lugar}

El trabajo experimental se llevó a cabo en el laboratorio de tecnología de la madera de la Estación Experimental Pucallpa del Instituto Nacional de Investigación Agraria

(INIA).

\section{Maderas}

El Cuadro 1 presenta la relación de maderas estudiadas.

\section{Cuadro 1. Relación de maderas estudiadas.}

\begin{tabular}{|l|l|l|}
\hline Nombre comun & Nombre científico & FAMILIA \\
\hline Atadijo & Trema micrantha & ANONACEAE \\
Caucho Masha & Sapium marmieri Hubert & MORACEAE \\
Huamanzamana & Jacaranda copaiba (Aubl) D. Don & BIGNONIACEAE \\
Topa & Ochroma piramydale & BOMBACEAE \\
\hline
\end{tabular}




\section{Procedencia de las maderas}

Las trozas provienen de un bosque secundario ubicado en la carretera Federico Basadre, km 18. El bosque tiene una extensión de 7706 ha y ha sido adjudicado inmediatamente después de rozado al Instituto Superior Tecnológico «Suiza».

\section{Número de trozas}

Se ha tomado tres árboles por especie y dos niveles por árbol. Se han marcado árboles coetáneos de estas especies para investigaciones complementarias sobre la influencia de la edad de los árboles en las propiedades tecnológicas de las maderas.

\section{Identificación dendrológica}

La identificación taxonómica se efectuó en muestras dendrológicas completas en el laboratorio de dendrología de la Facultad de Ciencias Forestales de la UNU.

\section{Probetas de madera}

Se ha utilizado probetas de madera de $2 \mathrm{~cm}$ x $2 \mathrm{~cm}$ x $40 \mathrm{~cm}$, simplemente aserradas, libres de defectos y signos de biodeterioro, acondicionadas en laboratorio hasta alcanzar el contenido de humedad de equilibrio.

\section{Repeticiones}

Se ha ensayado dos probetas por árbol de procedencia y nivel longitudinal (12 probetas por especie).

\section{Materiales}

Pentaclorofenol grado técnico.

Petróleo diessel 2.

Reactivo leuco base (ITINTEC 251.029).

Pintura epóxica con base de aluminio.

\section{Equipos}

Balanza electrónica $0,1 \mathrm{~g}$ de precisión. 
Estufa con termostato regulable.

Maquinaria de carpintería.

Tinas de inmersión.

Martillo.

Cuñas marcadoras.

\section{Procedimiento}

Los ensayos se efectuaron de acuerdo con las especificaciones propuestas por Aróstegui (1970) para ensayos de preservación por inmersión prolongada.

\section{RESULTADOS}

El Cuadro 2 presenta los resultados obtenidos en los ensayos de preservación de cuatro maderas por el método de inmersión prolongada con solución de pentaclorofenol al 5\% a temperatura ambiente.

\section{Cuadro 2. Características de preservación de cuatro maderas.}

\begin{tabular}{lrrrrr}
\hline Madera & \multicolumn{5}{c}{ Valor promedio } \\
& $\begin{array}{c}\text { Densidad } \\
\mathbf{g} / \mathrm{cm}^{3}\end{array}$ & $\begin{array}{c}\text { Absorción } \\
\mathrm{l} / \mathbf{m}^{3}\end{array}$ & $\begin{array}{c}\text { Retención } \\
\mathrm{Kg} / \mathbf{m}^{3}\end{array}$ & Penetración & Calificación \\
\hline Atadijo & $\mathbf{0 , 4 2}$ & 189 & $\mathbf{9 4 0}$ & Total regular & Fácil de tratar \\
Caucho masha & $\mathbf{0 , 4 8}$ & $\mathbf{5 0 5}$ & $\mathbf{2 5 , 2 0}$ & Total regular & Fácil de tratar \\
Huamanzamana & $\mathbf{0 , 4 0}$ & 410 & $\mathbf{2 0 , 5 0}$ & Total regular & Fácil de tratar \\
Topa & $\mathbf{0 , 1 8}$ & 148 & 7,40 & Total regular & Moderad. Tratable \\
\hline
\end{tabular}

\section{DISCUSION DE RESULTADOS}

De acuerdo con los resultados se tiene tres maderas fáciles de tratar por inmersión prolongada en solución de pentaclorofenol al 5\% y una es moderadamente tratable. En este grupo de maderas no se muestra ninguna tendencia a una relación inversamente proporcional entre la densidad básica y la absorción líquida. En efecto, la topa, que presenta una densidad muy baja, consecuencia de una gran cantidad de cavidades celulares o espacio libre para ser ocupado por la solución preservadora, tiene una absorción líquida mala. En contraposición. la madera de caucho masha, que tiene la mayor densidad básica del grupo de maderas ensayadas, demuestra tener una gran capacidad de absorción líquida. 
El comportamiento de la huamanzamana y caucho masha se debe principalmente a la estructura anatómica. Según la descripción anatómica efectuada por Aróstegui (1970) de ambas maderas y la de Valderrama et al. (1986) de la huamanzamana, ambas presentan porosidad difusa caracterizada por ser casi del mismo diámetro y estar uniformemente distribuida en la sección transversal de la madera. Aunque presentan pocos poros por unidad de superficie, éstos son de medianos a grandes, condiciones que aseguran una rápida absorción de la solución de tratamiento, como se observa en el Gráfico 1. También poseen, ambas maderas, fibras de pared delgada y lumen amplio, con capacidad para absorber líquidos. Aunque las fibras no son factores importantes en la absorción inicial de los líquidos, su permeabilidad relativa puede tener notable influencia en la extensión subsiguiente de los líquidos procedentes de los vasos. Esto puede explicar que la tendencia de decrecer la absorción líquida que se observa en todas las maderas ensayadas se invierta después de seis días de inmersión. Puede suponerse que los vasos se han saturado rápidamente y el líquido fluye lentamente a través de las fibras, desocupándose parte del espacio impregnado y permitiendo la absorción de más liquido.

Aun cuando la topa tiene una estructura anatómica similar a las anteriores, tal como la describen Aróstegui (1970) y Detienne (1983), la capacidad de impregnación es mucho menor. La penetración total observada indica que la solución de tratamiento se ha distribuido en toda la sección transversal; la absorción líquida baja indica que, aun cuando presenta poros grandes y fibras de amplio lumen, hay alguna sustancia que impide una mayor absorción. Este es un fenómeno frecuentemente observado en la topas una vez seca se comporta como un material impermeable.

Considerando los posibles usos de las maderas estudiadas, básicamente para usos interiores sin contacto con el suelo y de acuerdo con las especificaciones de la norma ITINTEC 251.035 (1970), es suficiente una retención de $4 \mathrm{~kg} / \mathrm{m}^{3}$, que es alcanzada por las cuatro maderas en los dos primeros días de tratamiento. Para asegurar una buena penetración y una adecuada distribución del preservador, puede ensayarse con soluciones a menor concentración y períodos de inmersión más breves. 


\section{CONCLUSIONES}

Mediante el método de tratamiento por inmersión prolongada en solución de pentaclorofenol al 5\% a temperatura ambiente, pueden obtenerse buenos resultados de absorción líquida y penetración en madera de Trema micrantha (atadijo), Sapium marmieri Hubert (caucho masha), Jacaranda copaia (Aubl.) D. Don (huamanzamana), calificándoseles como fáciles de tratar. En madera de Ochroma piramydale Swartz (topa) los resultados la ubican en la categoría de moderadamente tratable.

\section{BIBLIOGRAFIA}

AROStegui V., A. 1970. Estudio tecnológico de las maderas del Perú. Vol 1. Lima:UNA-MINAG. 104 pp.

— 1970. Estudio tecnológico de las maderas del Perú. Métodos y procedimientos. Vol II. Lima: UNA-MJNAG. 79 pp.

DETIENNE, P. et al. 1983. Estudio Anatómico do Lenho da familia bombacaceae do Amenia. En: Acta Amazónica XII (5-6): 83 1-868.

GONZALES F., R. 1970. Preservación de la madera. Lima: UNALM. 107 pp.

HIJNT, G. y GARRAT, A. 1962. Preservación de la madera. Barcelona: Salvat. $486 \mathrm{pp}$.

VALDERRAMA, H. et al. 1986. Estructura anatómica y clave de identificación de veinte especies forestales de la zona de Colonia Angamos, río Yavari y Jenaro Herrera. Iquitos: UNAP, IIAP. 